\title{
The Sum Capacity of Underlay Cognitive Broadcast Channel
}

\author{
Liang Li and Marius Pesavento \\ Communication Systems Group, Technische Universität Darmstadt, Darmstadt, Germany \\ $\{11$, m.pesavento\}@nt.tu-darmstadt.de
}

\begin{abstract}
We study the sum capacity of the underlay cognitive broadcast (BC) channel with $M$ primary receivers (PRs) and $K$ secondary receivers (SRs). In particular, the sum capacity is investigated under an average transmit power constraint (TPC) on the SRs and multiple average or peak interference power constraints (IPCs) on the PRs. In a fading environment, the average sum capacity is obtained from the solution of a convex optimization problem. The ergodic sum capacity $C$ is then derived from the limiting case where the number of fading state tends to infinity. Further, the asymptotic capacity is investigated in the homogeneous Rayleigh fading system for which two different asymptotic regimes are analyzed.
\end{abstract}

\section{INTRODUCTION}

The concept of cognitive radio (CR), first introduced by Mitola in 1998 [1], has been considered a promising path towards efficient spectrum usage. Recently, the CR found the way into standardization and practical systems implementations [2], [3]. Although the benefits of CR are intuitively appealing, many important questions regarding its capacity limits are still open. In [4], the recent results on the capacity of CR systems have been summarized. Depending on the chosen CR approach, the CR systems can be classified into three groups: the interweave, the underlay, and the overlay systems. In the interweave CR approach, the unlicensed cognitive users opportunistically access the radio resource: spectrum, time and space, complementary to the resources occupied by the licensed users. The underlay and overlay CR systems explicitly permit coexistence of cognitive and noncognitive transmissions and the adjunctive interference caused by the secondary users is allowed as long as it is harmless for the primary users. In underlay CR systems, radio access is granted to secondary devices only under the premise that the interference created at the primary users falls below some acceptable threshold. In the overlay CR systems, the secondary users facilitate the primary users' data transmission such that the primary users' communication does not deteriorate [4]-[6].

In this paper, our focus lies on the underlay CR approach. Our objective is to characterize the sum capacity of the underlay cognitive BC channel. We consider the single-input single-output (SISO) case where each transceiver is equipped with a single antenna. The sum capacity is analyzed under the average TPC and the average or peak IPCs. Applying the optimal user scheduling scheme, the maximal sum capacity averaged over a finite number of fading states can be formulated as the solution of the optimal power allocation problem. This problem is shown to be a convex and a unique solution and can be efficiently obtained using interior point methods.

Based on the results for the average sum capacity, the ergodic sum capacity $C$ is analyzed and its expressions for average and peak IPCs are derived. We then study the asymptotic behavior of the ergodic sum capacity for the cognitive BC channel. For simplicity we restrict our asymptotic analysis to the homogeneous Rayleigh fading system and consider the behaviors of $C$ in two asymptotic regimes. In the first asymptotic regime, we let $K \rightarrow \infty$ while fixing $M$ at a constant value. In the second asymptotic regime, we consider the case that $M \rightarrow \infty$ and $K$ is fixed as a constant.

\section{SySTEM MODEL}

Consider the underlay cognitive BC channel where a single ST sends independent information symbols to $K$ SRs by sharing a common narrow-band channel with $M$ PRs. All devices including the ST, the PRs, and the SRs, are equipped with a single antenna. The non-negative real-valued instantaneous channel power gains from the ST to the $k$ th SR and from the ST to the $m$ th PR are denoted by $h_{k}$ and $g_{m}$, respectively, for $1 \leq k \leq K$ and $1 \leq m \leq M$. Further, we denote the realizations of $h_{k}$ and $g_{m}$ at a particular fading state $i$ as $h_{k, i}$ and $g_{m, i}$, respectively, and assume that the ST has perfect channel knowledge of $h_{k, i}$ and $g_{m, i}$ at each fading state. Throughout this paper, we assume that the power gains are generated by stationary ergodic processes and that all power gains are mutually independent. For the computational tractability, each power gain is assumed to have a continuous and differentiable cumulative distribution function (cdf). The additive noise at the PRs and SRs is assumed to be independent circular symmetric zero-mean complex Gaussian with unit variance. We study the sum capacity of the cognitive $\mathrm{BC}$ channel under the constraints that the average transmit power does not exceed the available power budget $P$ and the average or peak interference temperature caused at the $m$ th PR falls below a given interference temperature threshold $Q_{m}$ or $\hat{Q}_{m}$, respectively.

Denoting the instantaneous transmit power at the $i$ th fading state as $p_{i}$, the instantaneous interference caused at the $m$ th PR is then equal to $g_{m, i} p_{i}$. Note that the interference only depends on the channel power gain of the $m$ th PR $g_{m, i}$ and the transmitted power $p_{i}$, but not on the scheduling policy of the secondary system. In a conventional BC fading channel without any PRs present, it has been shown in [8] that the capacity-maximizing user scheduling scheme is to select only the user with the highest instantaneous channel power gain for data transmission. Given a specific amount of transmitted power $p_{i}$ in the $i$ th fading state, the capacity achieving user selection scheme for the conventional $\mathrm{BC}$ channel is thus also optimal for the cognitive BC channel as different SRs scheduling schemes induce the same interference at each PR. In the sequel, we assume that the Gaussian codebook, which maximizes the sum capacity of a conventional BC fading channel, is used. Hence, the achievable sum capacity in the $i$ th fading state can be expressed as $C_{s}(i)=\log \left(1+\frac{\hat{h}_{i} p_{i}}{\mathcal{N}_{0}}\right)$, where $\hat{h}_{i}$ is the instantaneous power gain of the scheduled SR and $\mathcal{N}_{0}$ denotes the noise power [11].

\section{Sum CApacity Under AVERAge IPCS}

\section{A. Sum capacity averaged over a finite number of fading states}

Given a sequence of $N<\infty$ fading states $^{1}$, the average sum capacity $\bar{C}_{s}$ can be expressed as

$$
\bar{C}_{s}\left(p_{1}, \cdots, p_{N}\right)=\frac{1}{N} \sum_{i=1}^{N} C_{s}(i)=\frac{1}{N} \sum_{i=1}^{N} \log \left(1+\frac{\hat{h}_{i} p_{i}}{\mathcal{N}_{0}}\right) .
$$

\footnotetext{
${ }^{1}$ We assume $N \gg \max \{K, M\}$ since in the fading environment, the data
} transmission generally undergoes a large number of fading states. 
In this subsection we maximize $\bar{C}_{s}$ over $p_{i}(i=1, \cdots, N)$ under an average TPC and $M$ average IPCs. Defining $\mathbf{p}=\left[p_{1}, \cdots, p_{N}\right]^{T}$, the problem can be stated as

$$
\begin{array}{ll}
\max _{\mathbf{p} \in \mathbb{R}^{N}} & \frac{1}{N} \sum_{i=1}^{N} \log \left(1+\frac{\hat{h}_{i} p_{i}}{\mathcal{N}_{0}}\right) \\
\text { s. t. } & \bar{P} \triangleq \frac{1}{N} \sum_{i=1}^{N} p_{i} \leq P ; \\
& \bar{Q}_{m} \triangleq \frac{1}{N} \sum_{i=1}^{N} g_{m, i} p_{i} \leq Q_{m}, m=1, \cdots, M ; \\
& p_{i} \geq 0, i=1, \cdots, N,
\end{array}
$$

where (2b) and (2c) are the average TPC and average IPCs, respectively, $\bar{P}$ in (2b) denotes the consumed average transmit power and $\bar{Q}_{m}$ in (2c) is the induced average interference temperature at the $m$ th PR. The existence of a unique solution is assured by the following proposition.

Proposition 1: The optimization problem in (2) has a unique global maximum $\mathbf{p}^{\star}$.

Proof: Problem in (2) can be formulated as an equivalent minimization problem with a convex objective function. The feasible set of the problem in (2), given by the constraints (2b)-(2d), is a polyhedral set which is closed and convex. Further, it immediately follows from (2b) and (2d) that the feasible set of problem in (2) is bounded as $0 \leq p_{i} \leq N P$ for all $i=1, \cdots, N$, hence it is also compact. This feasible set is non-empty since $\mathbf{p}=\mathbf{0}_{N \times 1}$ is a feasible solution. Then, following the Weierstrass' Theorem [14, Proposition A.8], there exists a maximum $\mathbf{p}^{\star}$ of the objective function (2a). Moreover, the objective function (2a) is strictly concave since its Hessian is negative definite for all feasible p. Also, the objective function (2a) is proper since $-\infty<\bar{C}_{s}<\infty$ for all feasible p. By Proposition 2.1.2 in [13, p. 87], $\mathbf{p}^{\star}$ is the unique global maximum of $\bar{C}_{s}$ over the feasible set defined by (2b)-(2d).

The problem in (2) is a convex optimization problem with linear inequality constraints which can efficiently be solved using e.g. interior point methods [16]. Inserting the optimal power allocation $\mathbf{p}^{\star}$ in (1), the sum capacity $\bar{C}_{s}$ is obtained. In the following we derive an alternative procedure for computing the optimal power allocation, known as the constraint water-filling algorithm. Towards this aim we write the Lagrangian function of (2) as [14]

$$
\begin{aligned}
\mathcal{L}(\mathbf{p}, \boldsymbol{\mu}) & =-\frac{1}{N} \sum_{i=1}^{N} \log \left(1+\frac{\hat{h}_{i} p_{i}}{\mathcal{N}_{0}}\right)+\mu_{0}\left(\frac{1}{N} \sum_{i=1}^{N} p_{i}-P\right) \\
& +\sum_{m=1}^{M} \mu_{m}\left(\frac{1}{N} \sum_{i=1}^{N} g_{m, i} p_{i}-Q_{m}\right)-\sum_{j=M+1}^{M+N} \mu_{j} p_{j-M},
\end{aligned}
$$

where $\mu_{j}(j=0,1, \cdots, M+N)$ are the Lagrange multipliers. Let us define $\boldsymbol{\mu}=\left[\mu_{0}, \mu_{1}, \cdots, \mu_{M+N}\right]^{T}$ as the vector of Lagrange multipliers. Since all constraints in (2) are linear, from [14, Prop. 3.3.6 and 3.3.7], there exists a vector of optimal Lagrange multipliers $\boldsymbol{\mu}^{\star}$ satisfying the Karush-Kuhn-Tucker (KKT) necessary conditions [14, Proposition 3.3.1]

$$
\begin{gathered}
\nabla_{\mathbf{p}} \mathcal{L}\left(\mathbf{p}^{\star}, \boldsymbol{\mu}^{\star}\right)=\mathbf{0}_{N \times 1}, \boldsymbol{\mu}^{\star} \geq \mathbf{0}_{(M+1+N) \times 1} \\
\mu_{0}^{\star}\left(\frac{1}{N} \sum_{i=1}^{N} p_{i}^{\star}-P\right)=0,-\mu_{j}^{\star} p_{j-M}^{\star}=0 ; M+1 \leq j \leq M+N,(4 \mathrm{~b}) \\
\mu_{m}^{\star}\left(\frac{1}{N} \sum_{i=1}^{N} g_{m, i} p_{i}^{\star}-Q_{m}\right)=0 ; m=1, \cdots, M
\end{gathered}
$$

where (4b)-(4c) are commonly referred to as the complementary slackness (CS) conditions. Reformulating the KKT conditions (4a) and $(4 \mathrm{c})$, the optimal solution $\mathbf{p}^{\star}$ can be expressed as

$$
p_{i}^{\star}=\left[\frac{1}{\sum_{m=1}^{M} \mu_{m}^{\star} g_{m, i}+\mu_{0}^{\star}}-\frac{\mathcal{N}_{0}}{\hat{h}_{i}}\right]^{+} \text {for } i=1, \cdots, N,
$$

where $[x]^{+}$denotes the maximum of $x$ and 0 and the Lagrange multipliers $\mu_{m}^{\star}$ for $i=0, \cdots, M$ must be chosen such that the CS conditions in (4b) and (4c) are satisfied. A similar constrained water-filling solution as in (5) has been previously derived in [7] for the ergodic capacity of the cognitive BC channel.

Note that in a practical situation and for $N$ given fading states, the knowledge of all the channel power gains $g_{m, i}$ and $\hat{h}_{i}(m=$ $1, \cdots, M, i=1, \cdots, N)$ is required at the $\mathrm{ST}$ to compute the optimal power allocation $\mathbf{p}^{\star}$.

\section{B. Ergodic sum capacity under average IPCs}

The ergodic sum capacity is defined as the statistic expectation of the sum capacity with respect to the random channel power gains $g_{m}$ and $h_{k}, m=1, \cdots, M$ and $k=1, \cdots, K$. As we assume stationary ergodic channels, the ergodic sum capacity is equivalent to the average sum capacity in problem (2) for the limiting case of $N \rightarrow \infty$. The problem in (2) for $N \rightarrow \infty$ can be reformulated as

$$
\begin{aligned}
& C=\max _{p \geq 0} \mathcal{E}\left\{\log \left(1+\frac{\hat{h} p}{\mathcal{N}_{0}}\right)\right\} . \\
& \text { s. t. } \bar{P} \triangleq \mathcal{E}\{p\} \leq P ; \bar{Q}_{m} \triangleq \mathcal{E}\left\{g_{m} p\right\} \leq Q_{m}, 1 \leq m \leq M,
\end{aligned}
$$

where the transmit power $p$ is a random variable which according to (6) depends on the random channel power gains $g_{m}$ and $\hat{h}$.

We remark, that the Proposition 1 and the KKT conditions (4a)(4c) presented in the last subsection hold true for arbitrary values of $N$. Therefore, also in the limiting case of $N \rightarrow \infty$ the optimal power allocation in each fading state is given by (5). Hence, replacing the channel realizations $g_{m, i}$ and $\hat{h}_{i}$ in (5) by its corresponding random variables $g_{m}$ and $\hat{h}$, the optimal power allocation can be formulated as

$$
p^{\star}=\left[\frac{1}{\sum_{m=1}^{M} \mu_{m}^{\star} g_{m}+\mu_{0}^{\star}}-\frac{\mathcal{N}_{0}}{\hat{h}}\right]^{+},
$$

where $\mu_{m}^{\star} \geq 0$ with $m=0, \cdots, M$ are the optimal Lagrange multipliers satisfying the following CS conditions for $N \rightarrow \infty$

$$
\begin{gathered}
\mu_{0}^{\star}\left(\mathcal{E}\left\{p^{\star}\right\}-P\right)=0 ; \\
\mu_{m}^{\star}\left(\mathcal{E}\left\{g_{m} p^{\star}\right\}-Q_{m}\right)=0, \quad m=1, \cdots, M .
\end{gathered}
$$

It can be shown $[14, p .279]$ that the optimal solution $\mu^{\star}$ is unique if $\mathbf{p}^{\star}$ is regular, that is, if the gradients of all active inequality constraints are linearly independent at $\mathbf{p}^{\star}$. As all power gains are generated by stationary ergodic random processes, in the limiting case of $N \rightarrow \infty$, the number of strictly positive $p_{i}(i=1, \cdots, N)$ becomes much larger than $M+1$, i.e., the number of active constraints in problem (2) becomes much less than the dimension of each gradient vector, $N$. Thus, the gradients of all active constraints are almost sure to be linearly independent at $\mathbf{p}^{\star}$ in this case. This means that $\mathbf{p}^{\star}$ becomes regular and $\boldsymbol{\mu}^{\star}$ becomes unique as $N \rightarrow \infty$.

In contrast to the case of a finite number of fading states, problem (6) cannot be directly solved using interior point methods due to the statistical expectation involved. However, a unique solution can be obtained from the constrained water-filling solution (7) by first computing the optimal Lagrange multipliers from (8) and (9) and then inserting these values back into (7).

In the following, we derive a general procedure to efficiently compute the optimal Lagrange multipliers. The idea is to identify the inactive TPC and IPCs and discard them from the optimization problem. Given a particular budget $P$ at the transmitter and the average interference thresholds $Q_{m}(i=1, \cdots, M)$ for all $M$ PRs, we identify those PRs which undergo an interference temperature strictly lower than their thresholds. Similarly, we investigate if the entire transmit power budget is consumed by the optimal power allocation scheme. The following proposition provides a useful insight into this issue. 


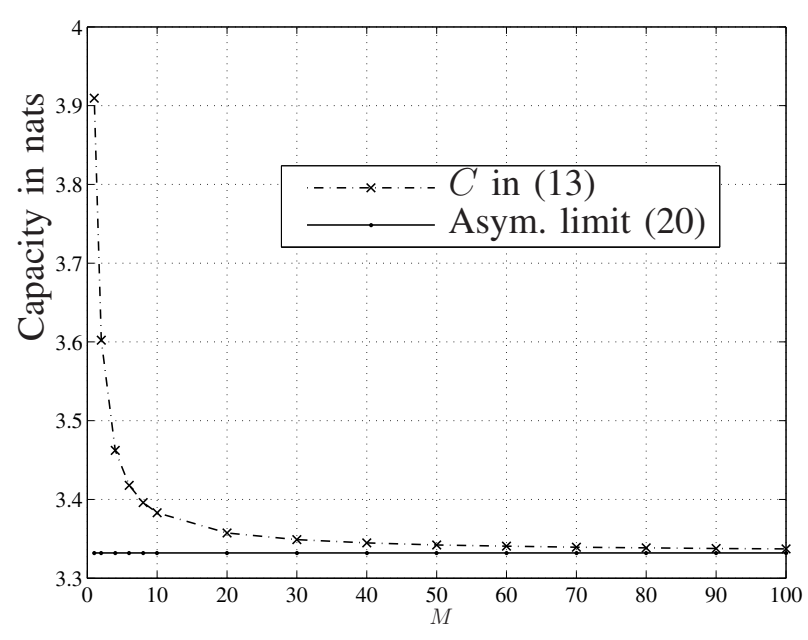

Fig. 1. Asymptotic behavior of $C$ under average IPCs, where $K=10$, $P=20 \mathrm{~dB}$ and $Q=10 \mathrm{~dB}$.

Proposition 2: With the optimal power allocation $p^{\star}$ given in (7), for any two PRs $k$ and $j$ with $\bar{Q}_{k}=Q_{k}$ and $\bar{Q}_{j}<Q_{j}$, we have $\frac{\bar{Q}_{k}}{\mathcal{E}\left\{g_{k}\right\}} \leq \bar{P}=\frac{\bar{Q}_{j}}{\mathcal{E}\left\{g_{j}\right\}}$.

The proof of Proposition 2 is omitted for brevity and will be present in the full version of this paper. Proposition 2 shows that any PR with an inactive IPC experiences an average interference power equal to its expected channel power gain multiplied by the average transmitted power. Similarly, for any PR with an active IPC, the average interference at that PR is less than or equal to its expected channel power gain multiplied by the average transmitted power.

Without loss of generality, let us order the $M$ PRs such that

$$
\frac{Q_{1}}{\mathcal{E}\left\{g_{1}\right\}} \leq \cdots \cdots \leq \frac{Q_{r}}{\mathcal{E}\left\{g_{r}\right\}} \leq P<\frac{Q_{r+1}}{\mathcal{E}\left\{g_{r+1}\right\}} \leq \cdots \leq \frac{Q_{M}}{\mathcal{E}\left\{g_{M}\right\}}
$$

where $0 \leq r \leq M$. Following the Proposition 2, we conclude that for the power allocation scheme $p^{\star}$ in (7), the IPCs in (6b) are inactive for all $r+1 \leq m \leq M$. Additionally, if the entire transmit power budget is consumed, i.e. $\bar{P}=P$, then the IPCs in (6b) are active for all $1 \leq m \leq r$, otherwise only a part of the IPCs with $1 \leq m \leq r$ are active. This observation shows that the PRs $r+1, \cdots, M$ have inactive IPCs and suffer interference temperatures strictly below their thresholds. According to the CS conditions in (9), their corresponding optimal Lagrange multipliers are all equal to zero. The optimal power allocation scheme $p^{\star}$ in (7) then becomes

$$
p^{\star}=\left[\frac{1}{\sum_{m=1}^{r} \mu_{m}^{\star} g_{m}+\mu_{0}^{\star}}-\frac{\mathcal{N}_{0}}{\hat{h}}\right]^{+},
$$

which is independent of $g_{m}$ with $r+1 \leq m \leq M$. Inserting (11) in (6a), we conclude that the ergodic sum capacity is also independent of $g_{m}$ with $r+1 \leq m \leq M$. Thus, the PRs $r+1, \cdots, M$ do not affect the ergodic sum capacity of the CR network.

The remaining Lagrange multipliers can then be computed by the following procedure.

- Initialization: Set $\mu_{l}^{\star}=0$ for $l=0, \cdots, M$ and $j=1$.

- Step $j$ (for $j=1, \cdots, r$ ): Compute $\mu_{m}$ with $m=1, \cdots, j$ by solving the following set of $j$ equations

$$
\mathcal{E}\left\{g_{i}\left[\frac{1}{\sum_{m=1}^{j} \mu_{m} g_{m}}-\frac{\mathcal{N}_{0}}{\hat{h}}\right]^{+}\right\}=Q_{i}, i=1, \cdots, j .
$$

If the obtained solution $p^{(j)}=\left[\frac{1}{\sum_{m=1}^{j} \mu_{m} g_{m}}-\frac{\mathcal{N}_{0}}{\hat{h}}\right]^{+}$is feasible, i.e., if $p^{(j)}$ satisfies the TPC and the IPCs in (6b), then set $\mu_{m}^{\star}=\mu_{m}$ for $m=1, \cdots, j$ and end the procedure; Otherwise go to the next step with $j=j+1$.

- Step $j=r+1$ : Calculate $\mu_{m}^{\star}$ for $m=0, \cdots, r$ by solving the following system of equations

$$
\begin{aligned}
& \mathcal{E}\left\{\left[\frac{1}{\sum_{m=1}^{r} \mu_{m}^{\star} g_{m}+\mu_{0}^{\star}}-\frac{\mathcal{N}_{0}}{\hat{h}}\right]^{+}\right\}=P \\
& \mathcal{E}\left\{g_{i}\left[\frac{1}{\sum_{m=1}^{r} \mu_{m}^{\star} g_{m}+\mu_{0}^{\star}}-\frac{\mathcal{N}_{0}}{\hat{h}}\right]^{+}\right\}=Q_{i}, i=1, \cdots, r .
\end{aligned}
$$

End the procedure.

Inserting the obtained $\mu_{m}^{\star}$ with $m=0, \cdots, r$ in (11), we obtain the optimal power allocation scheme $p^{\star}$. Substituting $p^{\star}$ for $p$ in (6a), the ergodic sum capacity can be expressed as

$$
C=\mathcal{E}\left\{\log \left(1+\frac{\hat{h}}{\mathcal{N}_{0}}\left[\frac{1}{\sum_{m=1}^{r} \mu_{m}^{\star} g_{m}+\mu_{0}^{\star}}-\frac{\mathcal{N}_{0}}{\hat{h}}\right]^{+}\right)\right\} .
$$

Given the probability density functions (pdfs) of $\hat{h}$ and $g_{m}$ ( $m=$ $1, \cdots, r)$, the ergodic sum capacity $C$ in the above equation can be easily computed numerically.

Note that if the statistical characteristics of the channel power gains are known, then the optimal Lagrange multipliers $\mu_{m}^{\star}(m=0, \cdots, r)$ in (13) can be computed offline. Thus, in a particular fading state, the ST can adjust its transmit power according to (11) solely based on the current channel states.

\section{Sum CAPACITY UNDER PEAK IPCS}

\section{A. Sum capacity averaged over $N$ fading states}

In this section, we maximize the average sum capacity $\bar{C}_{s}$ over $p_{i}(i=1, \cdots, N)$ under an average TPC and $M$ peak IPCs. This problem can be formulated similarly to problem (2) as

$$
\begin{array}{ll}
\max _{\mathbf{p} \in \mathbb{R}^{N}} & \frac{1}{N} \sum_{i=1}^{N} \log \left(1+\frac{\hat{h}_{i} p_{i}}{\mathcal{N}_{0}}\right) \\
\text { s. t. } & \bar{P} \triangleq \frac{1}{N} \sum_{i=1}^{N} p_{i} \leq P ; \\
& \tilde{Q}_{m} \triangleq \max _{i=1, \cdots, N}\left\{g_{m, i} p_{i}\right\} \leq \hat{Q}_{m}, m=1, \cdots, M ;(14 \mathrm{c}) \\
& p_{i} \geq 0, i=1, \cdots, N,
\end{array}
$$

where (14b) and (14c) are the average TPC and peak IPCs, respectively. $\bar{P}$ in $(14 \mathrm{~b})$ denotes the actually consumed average transmit power as defined in (2b) and $\tilde{Q}_{m}$ is the induced peak interference temperature at the $m$ th PR given in (14c)

Let us define $G_{i}=\max _{m=1, \cdots, M} \frac{g_{m, i}}{\hat{Q}_{m}}$ for $i=1, \cdots, N$. Then the peak IPCs in (14c) can be equivalently expressed as $G_{i} p_{i} \leq 1$ for $i=1, \cdots, N$. Similar to the problem (2), it can be proven that the optimization problem (14) has a unique global maximum $\mathbf{p}^{\star}$. This maximum can then be solved numerically using interior point methods [16]. By formulating the Lagrangian function and applying the KKT conditions, the optimal power allocation $\mathbf{p}^{*}$ can be expressed using the optimal Lagrange multipliers as

$$
p_{i}^{\star}=\frac{1}{N G_{i} \mu_{i}^{\star}-N \mu_{(i+N)}^{\star}+\mu_{0}^{\star}}-\frac{\mathcal{N}_{0}}{\hat{h}_{i}}, \text { for } i=1, \cdots, N .
$$

From the CS conditions, we observe that $\mu_{(i+N)}^{\star}=0$, if $p_{i}^{\star}>0$; and $\mu_{i}^{\star}=0$, if $G_{i} p_{i}^{\star}<1$, for $i=1, \cdots, N$. Hence, (15) can be compactly written as the water-filling type solution

$$
p_{i}^{\star}=\min \left\{\frac{1}{G_{i}},\left[\frac{1}{\mu_{0}^{\star}}-\frac{\mathcal{N}_{0}}{\hat{h}_{i}}\right]^{+}\right\} .
$$


The power allocation in (16) can also be derived from the Lagrange dual problem [7]. Using (16), we can distinguish two cases which facilitate the computation of the optimal power allocations. First, assume the case that $\frac{1}{N} \sum_{i=1}^{N} \frac{1}{G_{i}}>P$ for which we can prove by contradiction that $\bar{P}=P$. With $\bar{P}=P$ and the CS conditions, we can compute a unique solution of $\boldsymbol{\mu}^{\star}$ in this case. Next, assume that $\frac{1}{N} \sum_{i=1}^{N} \frac{1}{G_{i}} \leq P$. Then from (16) and exploiting the fact that the sum capacity increases with $p_{i}^{\star}$, we can conclude that the optimal power allocation is given by $p_{i}^{\star}=\frac{1}{G_{i}}$. The last observation outlines a simple procedure for computing the optimal power allocation for the case that $\frac{1}{N} \sum_{i=1}^{N} \frac{1}{G_{i}} \leq P$.

Similar to Section III-A, the optimal power allocation solution $\mathbf{p}^{\star}$ is computed based on the knowledge of all $\hat{h}_{i}$ and $g_{m, i}$ with $i=1, \cdots, N$ and $m=1, \cdots, M$.

\section{B. Ergodic sum capacity under peak IPCs}

Similar as in Section III-B, in this section we compute the ergodic sum capacity from the average sum capacity in problem (14) in the limiting case of $N \rightarrow \infty$. In the sequel, we use $G$ to denote the largest order statistic of $g_{m} / \hat{Q}_{m}$ with $m=1, \cdots, M$, i.e., $G=$ $\max _{m=1}, \cdots, M \frac{g_{m}}{\hat{Q}_{m}}$. We denote the cdf and pdf of $G$ by $F_{G}(x)$ and $f_{G}(x)$, respectively. Following the discussion presented in Subsection IV-A, the optimal power allocation scheme in (16) defines a random variable given by

$$
p^{\star}= \begin{cases}\frac{1}{G}, & \text { if } \mathcal{E}\left\{\frac{1}{G}\right\} \leq P ; \\ \min \left\{\frac{1}{G},\left[\frac{1}{\mu_{0}^{\star}}-\frac{\mathcal{N}_{0}}{h}\right]^{+}\right\}, & \text {otherwise. }\end{cases}
$$

Thus, in the case of $\bar{P}=\mathcal{E}\left\{\frac{1}{G}\right\} \leq P$, the ergodic sum capacity is given by $C=\mathcal{E}\left\{\log \left(1+\frac{\hat{h}}{\mathcal{N}_{0}} \frac{1}{G}\right)\right\}$.If $\mathcal{E}\left\{\frac{1}{G}\right\}>$ $P$, the ergodic sum capacity can be expressed as $C=$ $\mathcal{E}\left\{\log \left(1+\frac{\hat{h}}{\mathcal{N}_{0}} \min \left\{\frac{1}{G},\left[\frac{1}{\mu_{0}^{\star}}-\frac{\mathcal{N}_{0}}{\hat{h}}\right]^{+}\right\}\right)\right\}$, where the Lagrange multiplier $\mu_{0}^{\star}>0$ can be computed by solving the equation $\bar{P} \triangleq \mathcal{E}\left\{\min \left\{\frac{1}{G},\left[\frac{1}{\mu_{0}^{\star}}-\frac{\mathcal{N}_{0}}{\hat{h}}\right]^{+}\right\}\right\}=P$.

\section{Asymptotic Performance in RAyleigh FAding CHANNEL}

The results presented in Section III and IV are valid for any particular fading distribution. In this section, we consider the special case of Rayleigh fading channels and study the asymptotic behavior of the ergodic sum capacity. While in the first asymptotic regime we fix the number of PRs $M$ and let the number of SRs $K$ increase to infinity, in the second regime we fix $K$ and let $M$ increase to infinity. For computational tractability, we assume a Rayleigh faded homogeneous system, in which:

(A1) The channel gains of all PRs and SRs are independent and identically distributed (i.i.d.).

(A2) The pdf of the channel power gains $g_{m}$ and $h_{k}(m=$ $1, \cdots, M ; k=1, \cdots, K)$ is given by $f(x)=\exp (-x)$.

(A3) The PRs' interference thresholds are identical and equal to $Q$ and $\hat{Q}$ in the average and peak IPCs case, respectively.

Note that the particular choice of pdf in (A2) corresponds to Rayleigh fading channels with unit variance. Further, we note that the pdf of $\hat{h}$, i.e., the largest order statistic of $h_{k}$, is given by $f_{\hat{h}}(y)=$ $K\left(1-e^{-y}\right)^{K-1} e^{-y}[15]$.

\section{A. Asymptotic capacity for a large number of SRs}

Considering that the underlay cognitive $\mathrm{BC}$ channel benefits from the multiuser diversity advantage, we expect a capacity increase with the number of SRs. In particular, we are interested in the scaling order of the ergodic sum capacity as a function of the number of SRs $K$.
1) Average IPCs: We derive upper and lower bounds to investigate the asymptotic behavior of $C$ defined in (13). Note first that the ergodic sum capacity is a monotonically non-increasing function of the number of PRs $M$. Thus, the ergodic sum capacity in the case of $M=1$, which we denote as $\left.C\right|_{M=1}$, marks an upper bound for the case of any $M \geq 1$. A lower bound can be obtained from a feasible, but suboptimal constant power allocation scheme $p=p_{0}$, where the constant transmit power is characterized by $p_{0}=\min \left\{P, \frac{Q_{m}}{\mathcal{E}\left\{g_{m}\right\}}\right\}$ for $m=1, \cdots, M$. We denote this lower bound as $\left.C\right|_{p=p_{0}}$. Then, we have $\left.C\right|_{p=p_{0}} \leq C \leq\left. C\right|_{M=1}$. The fact that $\left.C\right|_{p=p_{0}}$ scales like $\log \log K$ for $K \rightarrow \infty$ can be straightforwardly deduced from the results in [9]-[10], where it has been shown that in the case of a constant transmit power, the sum capacity asymptotically scales as $\log \log K$. In [12], we have proven that the upper bound $\left.C\right|_{M=1}$ also increases with $\log \log K$ as $K \rightarrow \infty$. Hence, the ergodic sum capacity $C$ scales as $\log \log K$ for a large number of SRs $K$.

2) Peak IPCs: Similarly to the case of average IPCs, we derive upper and lower bounds to show the asymptotic behavior of the ergodic sum capacity $C$ under the peak IPCs. With the assumptions (A1)-(A3), the pdf of $g_{m} / \hat{Q}$ with $m=1, \cdots, M$ is given by $f_{g_{m} / \hat{Q}}(x)=\hat{Q} \exp (-\hat{Q} x)$. The pdf of $G$ can then be derived as $f_{G}(x)=M \hat{Q}\left(1-e^{-\hat{Q} x}\right)^{M-1} e^{-\hat{Q} x}$ and $\mathcal{E}\{G\}=$ $\frac{M}{\hat{Q}} \sum_{k=0}^{M-1}\left(\begin{array}{c}M-1 \\ k\end{array}\right) \frac{(-1)^{k}}{(k+1)^{2}}$. Due to the singularity at $G=0$, the expectation of $\frac{1}{G}$ in the Rayleigh fading case can be expressed in terms of the Cauchy principal value as

$$
\mathcal{E}\left\{\frac{1}{G}\right\}=\left\{\begin{array}{r}
\lim _{\epsilon \rightarrow 0^{+}}-\hat{Q} \operatorname{Ei}(-\hat{Q} \epsilon)=\infty, M=1 \\
\hat{Q} M \sum_{k=1}^{M-1}\left(\begin{array}{c}
M-1 \\
k
\end{array}\right)(-1)^{k+1} \log (k+1), M \geq 2 .
\end{array}\right.
$$

Making use of Jensen's inequality, we can find upper and lower bounds to show that $C$ increases as $\log \log K$ for large $K$. The detailed derivations are straightforward and omitted here for brevity.

\section{B. Asymptotic capacity for a large number of PRs}

The number of IPCs increases with $M$ while the objective functions in the capacity maximization problems (2), (6) and (14) are independent of $M$. Thus, the ergodic sum capacity is expected to be a non-increasing function of $M$ in this asymptotic regime. We are interested in the asymptotic limits of $C$ as $M \rightarrow \infty$.

1) Average IPCs: In the assumed homogeneous system, the $M$ IPCs in problem (6) are symmetric, which means that all $M$ IPCs are identical up to the ordering of the i.i.d random channel power gains $g_{m}$ with $m=1, \cdots, M$. Hence, in the sequel, we use $\mathcal{E}\{g\}$ to denote their expectations. If $P<\frac{Q}{\mathcal{E}\{g\}}$, then from the discussion in section III-B, all PRs can be omitted in the capacity analysis and the optimal power allocation scheme is given by the conventional water-filling solution. In this case, the ergodic sum capacity is given by $C=\int_{0}^{\infty} \log \left(1+\frac{y}{\mathcal{N}_{0}}\left[\frac{1}{\lambda_{0}}-\frac{\mathcal{N}_{0}}{y}\right]^{+}\right) f_{\hat{h}}(y) d y$, where $\lambda_{0}$ solves the equation $P=\int_{0}^{\infty}\left[\frac{1}{\lambda_{0}}-\frac{\mathcal{N}_{0}}{y}\right]^{+} f_{\hat{h}}(y) d y$. Obviously, $C$ remains constant in this case as $M$ increases. In the case of $P \geq \frac{Q}{\mathcal{E}\{g\}}$, the optimal power allocation scheme $p^{\star}$ becomes $p^{\star}=\left[\frac{1}{\mu^{\star} \sum_{m=1}^{M} g_{m}+\mu_{0}^{\star}}-\frac{\mathcal{N}_{0}}{\hat{h}}\right]^{+}$, where $\mu_{m}^{\star}=\mu^{\star}$ for all $m=1, \cdots, M$ due to the symmetry of the homogeneous system. Defining a new random variable as $S=\sum_{m=1}^{M} g_{m}$ and summing up the IPCs in (6b) for $m=1, \cdots, M$, we obtain an equivalent IPC which depends on the new random variable $S$

$$
M \bar{Q} \triangleq \int_{0}^{\infty} \int_{0}^{\infty} x\left[\frac{1}{\mu^{\star} x+\mu_{0}^{\star}}-\frac{\mathcal{N}_{0}}{y}\right]^{+} f_{S}(x) f_{\hat{h}}(y) d x d y=M Q
$$

where $\bar{Q}$ is the induced interference temperature at each $\mathrm{PR}$ and $f_{S}(x)$ is the pdf corresponding to $S$. Note that the equality $\bar{Q}=Q$ holds since $P \geq Q / \mathcal{E}\{g\}$. 
From the central limit theorem (CLT), the distribution of $(S-$ $M \mathcal{E}\{g\}) /\left(\sqrt{M} \sigma_{g}\right)$ approaches the standard normal distribution for a large $M$, i.e. $X=\frac{S-M \mathcal{E}\{g\}}{\sqrt{M} \sigma_{g}} \stackrel{d}{\rightarrow} \mathcal{N}(0,1)$, where $\sigma_{g}$ is the standard deviation of $g_{m}(m=1, \cdots, M)$ and " $\stackrel{d}{\rightarrow}$ " denotes the convergence in distribution. Then, the equivalent IPC in (18) can be rewritten as

$$
\begin{aligned}
\frac{Q}{\mathcal{E}\{g\}} & \simeq \mathcal{E}_{\hat{h}}\left\{\int_{-M^{\frac{1}{3}}}^{M^{\frac{1}{3}}}\left[\frac{1}{M \mu^{\star} \mathcal{E}\{g\}+\mu_{0}^{\star}}-\frac{\mathcal{N}_{0}}{\hat{h}}\right]^{+} \phi(x) d x\right\} \\
& \simeq \mathcal{E}_{\hat{h}}\left\{\left[\frac{1}{M \mu^{\star} \mathcal{E}\{g\}+\mu_{0}^{\star}}-\frac{\mathcal{N}_{0}}{\hat{h}}\right]^{+}\right\},
\end{aligned}
$$

where $\phi(x)$ is the pdf of the standard Gaussian distribution of zero mean and unit variance. The approximations hold for large values of $M$ since $\phi(x) \simeq 0$ for $x<-M^{\frac{1}{3}}$ or $x>M^{\frac{1}{3}}$ and $\frac{\sigma_{g} x}{\mathcal{E}\{g\} \sqrt{M}}+1 \simeq 1$ for $-M^{\frac{1}{3}} \leq x \leq M^{\frac{1}{3}}$. Defining $\lambda_{0}^{\star}=$ $M \mu^{\star} \mathcal{E}\{g\}+\mu_{0}^{\star}$, we can rewrite the equivalent IPC in (18) as $\frac{Q}{\mathcal{E}\{g\}} \bumpeq$ $\int_{0}^{\infty}\left[\frac{1}{\lambda_{0}^{\star}}-\frac{\mathcal{N}_{0}}{y}\right]^{+} f_{\hat{h}}(y) d y$, and the value of $\lambda_{0}^{\star}$ can be obtained by solving this equation numerically. Similarly, for large $M \rightarrow \infty$, the TPC can be rewritten as $\bar{P} \simeq \int_{0}^{\infty}\left[\frac{1}{\lambda_{0}^{\star}}-\frac{\mathcal{N}_{0}}{y}\right]^{+} f_{\hat{h}}(y) d y \leq P$ and the ergodic sum capacity becomes

$$
C \backsim \int_{0}^{\infty} \log \left(1+\frac{y}{\mathcal{N}_{0}}\left[\frac{1}{\lambda_{0}^{\star}}-\frac{\mathcal{N}_{0}}{y}\right]^{+}\right) f_{\hat{h}}(y) d y,
$$

for $M \rightarrow \infty$. From the above discussion, we can see that for $P \geq$ $\frac{Q}{\mathcal{E}\{g\}}$ and $M \rightarrow \infty$, the ergodic sum capacity $C$ approaches to that of a conventional $\mathrm{BC}$ with an average transmit power $\frac{Q}{\mathcal{E}\{g\}}$. This fact is demonstrated by the Fig. 1, where as $M$ increases, $C$ in (13) asymptotically approaches the limit given by (20).

It should be noted that the analysis in this subsection is based on the homogeneity assumption (A1) and (A3). The Rayleigh fading assumption (A2) is not needed to derive the asymptotic capacity limits. Hence, the above asymptotic analysis is also valid for other fading types.

2) Peak IPCs: Let us next consider the asymptotic behavior of the ergodic sum capacity under assumptions (A1)-(A3). Using the Jensen's inequality, an upper bound on $C$ can be obtained as $C \leq \mathcal{E}_{\hat{g}}\left\{\log \left(1+\frac{a}{\hat{g}}\right)\right\}$, where $a=\hat{Q} \mathcal{E}\{\hat{h}\} / \mathcal{N}_{0}$ is a constant and $\hat{g}=\max _{m=1, \cdots, M} g_{m}$. As $M$ increases, $X=\hat{g}-\log M$ has a limiting distribution whose cdf is given by $F_{X}(x)=\exp \left(-e^{-x}\right)$ with $-\infty<x<\infty$. The probability that $|X|<(\log M)^{\frac{1}{2}}$ for a large value of $M$ can be expressed as $\operatorname{Pr}\left(|X| \leq(\log M)^{\frac{1}{2}}\right)=$ $F_{X}\left((\log M)^{\frac{1}{2}}\right)-F_{X}\left(-(\log M)^{\frac{1}{2}}\right) \simeq 1$ for $M \rightarrow \infty$. Thus, it is almost sure that $\log M-(\log M)^{\frac{1}{2}} \leq \hat{g} \leq \log M+(\log M)^{\frac{1}{2}}$ as $M$ increases. Since $(\log M)^{\frac{1}{2}} \ll \log M$ for $M \rightarrow \infty$, the upper bound asymptotically scales with

$$
\tilde{C}=\log \left(1+\frac{a}{\log M}\right) .
$$

Thus, both $\tilde{C}$ and $C$ asymptotically converges to 0 as $M \rightarrow \infty$.

\section{CONCLUSIONS}

We have investigated the fundamental limits of sum capacity in the underlay $\mathrm{CR}$ BC channel. In the case of a finite number of fading states, the sum capacity maximization problem forms a convex optimization problem, which has a unique solution. As the number of fading states approaches infinity, the average sum capacity asymptotically converges to the ergodic sum capacity $C$. We derived expressions for the sum capacity under both average and peak
IPCs. These expressions were then used to determine the asymptotic behaviors of $C$ in the Rayleigh fading case.

\section{REFERENCES}

[1] J. Mitola, "Cognitive radio: An integrated agent architecture for software defined radio," Ph.D dissertation, KTH, Stockholm, Sweden, 2000.

[2] C. Cordeiro, K. Challapali and D. Birru, "IEEE 802.22: An introduction to the first wireless standard based on cognitive radios," Journal of Commun., vol. 1, no. 1, pp. 38-47, April 2006.

[3] C. Stevenson, G. Chouinard, Z. Lei, W. Hu, S. Shellhammer, and W. Caldwell, "IEEE 802.22: The first cognitive radio wireless regional area network standard," IEEE Commun. Magazine, vol. 47, pp. 130-138, Jan. 2009.

[4] A. Goldsmith, S. A. Jafar, and S. Srinivasa, "Breaking spectrum gridlock with cognitive radios: an information theoretic perspective," Proceedings of the IEEE, vol. 97, pp. 894-914, May 2009.

[5] N. Devroye, P. Mitran, V. Tarokh, "Achievable rates in cognitive radio channels," IEEE Trans. Inf. Theory, vol. 52, no. 5, pp. 1813-1827, May 2006.

[6] L. Li, F. A. Khan, M. Pesavento, and T. Ratnarajah, "Power allocation and beamforming in overlay cognitive radio systems," in Proc. IEEE VTC, Budapest, Hungary, accepted, May 2011.

[7] R. Zhang, S. Cui, and Y. C. Liang, "On ergodic sum capacity of fading cognitive multiple-access and broadcast channels," IEEE Trans. Inf. Theory, vol. 55, no. 11, pp. 5161-5178, Nov. 2009.

[8] D. N. C. Tse, "Optimal power allocation over parallel Gaussian broadcast channels," in Proc. ISIT'97, Ulm, Germany, p. 27, June 1997.

[9] M. Sharif and B. Hassibi, "On the capacity of MIMO broadcast channels with partial side information," IEEE Trans. Inf. Theory, vol. 51, no. 2 , pp. 506-522, Feb. 2005.

[10] S. Sanayei and A. Nosratinia, "Opportunistic downlink transmission with limited feedback," IEEE Trans. Inf. Theory, vol. 53, pp. 4363-4372, Nov. 2007.

[11] T. M. Cover and J. A. Thomas, Elements of Information Theory, John Wiley \& Sons, 1991.

[12] L. Li, M. Pesavento, and A. B. Gershman, "On ergodic sum capacity of underlay cognitive broadcast channels," in Proc. PIMRC'2010, Istanbul, Turkey, accepted, Sep. 2010.

[13] D. P. Bertsekas, A. Nedic, and A. E. Ozdaglar, Convex Analysis and Optimization, Belmont, MA: Athena Scientific, 2003.

[14] D. P. Bertsekas, Nonlinear Programming, Second Edition, Belmont, MA: Athena Scientific, 1999.

[15] H. A. David and H. N. Nagaraja, Order Statistics, John Wiley \& Sons, 2003.

[16] S. Boyd and L. Vandenberghe, Convex Optimization, Cambridge University Press, 2004. 